\title{
Superficial Temporal Vessels for Head and Scalp Microsurgical Reconstruction
}

\author{
Andrea Vicente-Pardo, MD ${ }^{1}$ Alberto Pérez-García, MD ${ }^{1}$ Jorge Balaguer-Cambra, MD ${ }^{1}$ \\ Alessandro Thione, MD ${ }^{1}$ Alberto Sánchez-García, MD ${ }^{1}$
}

1 Department of Plastic Surgery and Burns, University and Polytechnic Hospital La Fe Valencia, Valencia, Spain

J Reconstr Microsurg Open 2020;5:49.

We have read with interest the article entitled "Utilization of Intraparotid Segments of Superficial Temporal Vessels (STV) for Head and Scalp Free Flap Micronastomosis: A Clinical, Histological, and Cadaveric Study." We congratulate Venkatesh et al for their relevant work. Superficial temporal vessels (STV) are very suitable vessels for head and scalp reconstruction $^{2}$ due to their proximity and relative easy dissection. Because of their tortuous anatomy and their small diameter, STV have been historically considered to be prone to vasospasm, and not appropriate vessels for microanastomosis in free flap reconstruction. In their article, authors explain techniques to facilitate their dissection and make it more reliable.

We would like to share our positive experience with these receptor vessels. At our hospital, STV are considered as primary recipient vessels in microsurgery reconstruction of the scalp and upper and middle thirds of the face. Between 2015 and 2020, 16 patients underwent head-free flap reconstruction in our department, utilizing STV for anastomosis. Preauricular segment of STV were used as recipient vessels in all the cases. Average age was 54.9 and 10 patients had prior radiation (62.5\%). Defect etiology included surgical wound infection or necrosis (50\%), tumor resection (25\%), osteoradionecrosis (12.5\%), arteriovenous malformation (6.25\%), and facial lipoatrophy (6.25\%). Flaps transferred included radial forearm (31.25\%), anterolateral thigh (25\%), latissimus dorsi (18.75\%), gracilis (18.75\%), and dorsalis pedis (6.25\%).

Temporal artery trunk bifurcates into frontal and parietal branches (which have different calibers), so it is possible to choose the best matching vessel with the flap artery. This
Address for correspondence Alberto Pérez-García, MD, Department of Plastic Surgery and Burns, La Fe University Hospital, Avinguda de Fernando Abril Martorell, no. 10646026 Valencia, Spain (e-mail: albertoperezgarci@gmail.com).

occurs few millimeters above preauricular segment. Although there is only one comitant vein, since it has few valves and many communications, it can be used in both directions, allowing two venous anastomosis. Although vasospasm could occur after artery is cut, we usually dilate vessels mechanically and irrigate local vasodilators. Our result is that no anastomosis revisions were required. In our series, there were no instances of postoperative complications including operative takeback, partial/total necrosis, or facial nerve injury.

We agree with the authors that STV are the first-choice option for upper and middle face and scalp reconstruction, due to their anatomical location, and even though vasospasm sometimes happened during the dissection, it has not had relevant clinical consequences (anastomosis revision or flap loss) in our series.

Conflict of Interest

None declared.

\section{References}

1 Venkatesh V, Fracol M, Turin S, Ellis M, Alghoul M. Utilization of intraparotid segments of superficial temporal vessels for head and scalp free flap microanastomosis: a clinical, histological, and cadaveric study. J Reconstr Microsurg 2020;36(04):253-260

2 Hansen SL, Foster RD, Dosanjh AS, Mathes SJ, Hoffman WY, Leon P. Superficial temporal artery and vein as recipient vessels for facial and scalp microsurgical reconstruction. Plast Reconstr Surg 2007; 120(07):1879-1884
DOI https://doi.org/ 10.1055/s-0040-1715583. ISSN 2377-0813.
Copyright (c) 2020 by Thieme Medical Publishers, Inc., 333 Seventh Avenue, New York, NY 10001, USA. Tel: +1(212) 760-0888.
License terms c) $(1) \triangleq$ 